\title{
Advanced characterization of by-product carbon film obtained by thermal chemical vapor deposition during CNT manufacturing
}

\author{
Aikaterini-Flora A. Trompeta, Elias P. Koumoulos, Ioannis A. Kartsonakis, and Costas A. Charitidis* \\ RNanolab, School of Chemical Engineering, National Technical University of Athens, Heroon Polytechneiou Street, Zografos, \\ Athens 15780, Greece
}

Received 9 December 2016 / Accepted 23 February 2017

\begin{abstract}
This study is focused on the investigation of the morphology and the characteristics of carbon films, obtained by thermal chemical vapor deposition (T-CVD) on different substrates, during carbon nanotubes (CNTs) manufacturing. These films were examined, by means of optical and electronic microscopy, X-ray photoelectron spectroscopy and Raman spectroscopy. Additional measurements were conducted related with the estimation of their nanomechanical properties. The topography is studied, in combination with the assessment of their mechanical behavior at nanoscale. Through nanoindentation, hardness and elastic modulus together with wear resistance index $(H / E)$ were evaluated. According to this study, it may be remarked that the obtained film is classified as an amorphous carbon film.
\end{abstract}

Key words: Carbon coatings, Thin films, Chemical vapor deposition, Advanced characterization, Nanomechanical properties

\section{Introduction}

Carbon coatings and nanostructures (amorphous hydrogenated, a-C:H) reveal an increased amount of interest during the last years due to their unique properties, such as enhanced wear resistance and hardness, optical transparency and low friction coefficients [1, 2]. Amorphous carbon films and 2D carbon nanostructures are widely used in coatings with anticorrosion properties, in carbon-based semiconductors [3] or super capacitors [4] and as coatings on microspheres for inertial confinement targets [5]. Another application that worth mentioning is the use of amorphous carbon based materials as antireflective coatings on crystalline silicon solar cells, which has been utilized by da Silva et al. [6], using plasma enhanced chemical vapor deposition (PECVD) and the filtered cathodic vacuum arc technique. In the field of microelectronics, amorphous carbon films have gained attraction for their use in microelectromechanical systems (MEMS), as they can increase their performance due to their interesting mechanical properties [7]. Amorphous carbon films have potential applications also in the field of biomaterials, especially during cardiovascular surgeries, due to the high importance of a low blood surface interaction [8]. The aforementioned routes of utilization give a straight reason for the investigation of such films, with a thorough characterization of the manufactured material.

*e-mail: charitidis@chemeng.ntua.gr
The production of carbon and carbon-based thin films can be accomplished via several physical and chemical vapor deposition (CVD) methods such as magnetron sputtering, plasma jet CVD and plasma enhanced CVD (PECVD). Despite the fact that excellent quality a-C:H films are obtained from the most of these techniques, the films growth rate is not sufficient. Regarding all the aforementioned techniques, it may be remarked that carbon coatings can be easily synthesized via thermal CVD (T-CVD), due to the fact that the growth of the film is affected by two controllable parameters; the flow rate of the feedstock and the reaction temperature. However, it should be noticed that the produced a-C:H films exhibit enhanced diffusion of carbon into the substrate and often the films suffer of high residual stresses. These two drawbacks restrict the $\mathrm{a}-\mathrm{C}: \mathrm{H}$ films of full commercialization due to poor adhesion to the substrates. Other alternatives of CVD have also been employed for the manufacturing of amorphous carbon films. Specifically, Mitura et al. [9] have achieved the manufacturing of amorphous carbon layers by r.f. dense plasma CVD. A patent also exists regarding the forming of amorphous carbon films by the vaporization of liquid hydrocarbon compounds and their ionization, for the manufacturing of semiconductor devices [10].

Numerous are the cases in which the manufacturing of a material can lead to avoided or maybe useful by-products. This is mainly evident in nano-manufacturing processes, where extreme conditions are necessary for the synthesis of unique 
materials in nano-dimensions [11]. Most of the time is difficult to direct a reaction only to one way and obtain a desired product without any impurities or side products. Especially, regarding carbon and its numerous allotropic structures, the manufacturing of a specific nanostructure, such as carbon nanotubes (CNTs) may be challenging. A common example is the difficulty of separation between CNTs and carbon nanofibers (CNFs) [12]. In some cases, CNTs also coexist with amorphous carbon, soot or even fullerenes but, be that as it may, these structures resemble macroscopically and they appear as a black powder. There are also cases, that amorphous carbon layers can be used as catalysts for a metal-free growth of CNTs, as has been reported by Jae Keun et al. [13]. The same catalytic behavior was evident by Bachmatiuk et al. [14] for the growth of vertical graphene, using oxidizing gases. Yang et al. have achieved the synthesis of ultrathin carbon films, consisting of few short, curved graphene layers and thin amorphous carbon films [15]. Finally, a novel application of the amorphous carbon films is their transformation into graphene, by current induced annealing, which opens up new avenues for bottom-up engineering of graphene-based devices [16].

Taking into the above raised issues concerning amorphous films manufacturing, it is critical to investigate extensively the structure and the circumstances that led to its synthesis. Let alone the explicit properties that may be revealed and the potential applications in which it can find use, which can shift it form a side-product to a main engineered manufactured material. Thus, the aim of this study is to identify and characterize a carbon film that appears during the CNT synthesis, using a T-CVD system. The carbon film has been noticed on different substrates inside the reactor (monocrystalline silicon wafer, ceramic boats made from $\mathrm{Al}_{2} \mathrm{O}_{3}$, quartz and so on). In order to specify the exact conditions that contributed for its synthesis, experiments with different parameters, substrates and in the presence or absence of catalyst took place. Several characterization techniques were performed, in order the obtained material to be fully characterized. Among others, Raman scattering is known to be a unique characterization tool for carbon-based materials, such as graphite, diamond, graphene and nanostructured carbons [17]. This technique was conducted synergistically, with X-ray photoelectron spectroscopy (XPS) and scanning electron microscopy (SEM), coupled with X-ray energy dispersive spectroscopy (EDS) to obtain a complete analysis of the film. Moreover, nanomechanical testing through nanoindentation has been employed both for the characterization of the films structural integrity and its classification.

\section{Materials and methods}

\subsection{Synthesis procedure}

The synthesis of the carbon coatings has been performed by T-CVD, using two hydrocarbon precursors; one in a solid state and one in a gaseous state. In both cases, carbon coatings have been deposited on different substrates, as it will be explained in the following. The T-CVD reactor consists of a

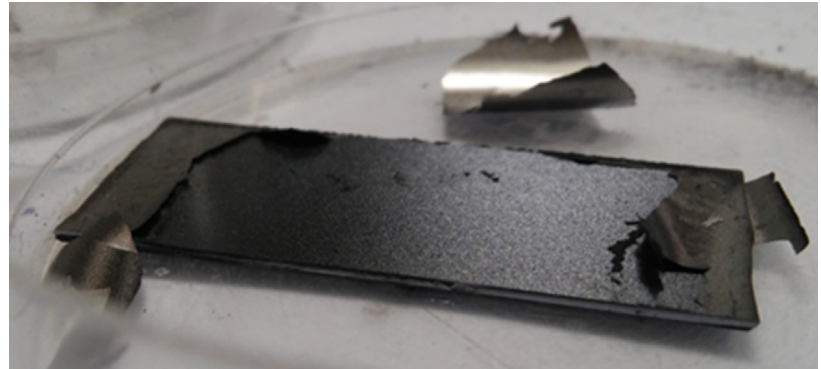

Figure 1. Carbon coating grown on Si wafer substrate.

tubular furnace, in which a quartz tube is placed. The quartz tube is connected with an inlet, from which inert nitrogen gas can pass, carrying the vapors of the carbon source.

As far as the solid precursor is concerned, it comprises by camphor ( $96 \%$ purity in weight, Sigma Aldrich), which is evaporated and carried by nitrogen $(300 \mathrm{~mL} / \mathrm{min})$ into the CVD reactor, which reserves a temperature of $900-960{ }^{\circ} \mathrm{C}$. The deposition time can last from $45 \mathrm{~min}$ to $2 \mathrm{~h}$, which depends on the time needed for the evaporation of the precursor. The carbon film is deposited on $\mathrm{Si}$ wafers $(\mathrm{P} /$ Boron $<100>$ ), paced in the middle of the isothermal zone of the CVD-reactor. However, the formation of the carbon films is evident also along the sidewalls of the quartz tube.

Concerning the gaseous precursor, acetylene can be used due its easy pyrolysis at temperatures from $500{ }^{\circ} \mathrm{C}$ [18]. In this case, the deposition takes place at lower temperatures, between $700{ }^{\circ} \mathrm{C}$ and $850^{\circ} \mathrm{C}$, and it lasts for $1 \mathrm{~h}$. As substrate, copper oxide nanoparticles can be used, placed on a ceramic boat, made from $\mathrm{Al}_{2} \mathrm{O}_{3}$ and $\mathrm{SiO}_{2}$. As in the previous case, carbon films are deposited on the quartz tube sidewalls, too.

\subsection{Characterization techniques}

Carbon coatings have been examined using an optical Microscope Axio Imager.A2m with four objective lenses $(20 \times, 40 \times, 50 \times$ and $100 \times)$ in reflective mode. Multiple images have been acquired and combined (z-stitching) for high depth of field (DOF) images, using Zerene Stacker software. Surface topography has been also studied by scanning electron microscopy using a FEI electron microscope.

Raman spectra were collected with a Renishaw inVia reflex Raman microscope. Measurements were carried out using a solid state $532 \mathrm{~nm}$ laser as excitation source, at room temperature, focused on the sample by means of 5, 20 and 100 objective lenses. XPS was conducted using aThermo Scientific K-Alpha XPS system (Thermo Fisher Scientific, UK) equipped with a micro-focused, monochromatic AlKa X-ray source $(1486.6 \mathrm{eV})$ with an energy resolution of $0.8 \mathrm{eV}$ and a pass energy of $15 \mathrm{eV}$.

The nanomechanical properties of the coatings have been tested with a Hysitron TriboLab ${ }^{\circledR}$ Nanomechanical Test Instrument, which allows the application of loads from $1 \mu \mathrm{N}$ to $30 \mathrm{mN}$ and records the displacement as a function of applied loads with a high load resolution $(1 \mathrm{nN})$ and a high displacement resolution $(0.04 \mathrm{~nm})$. 

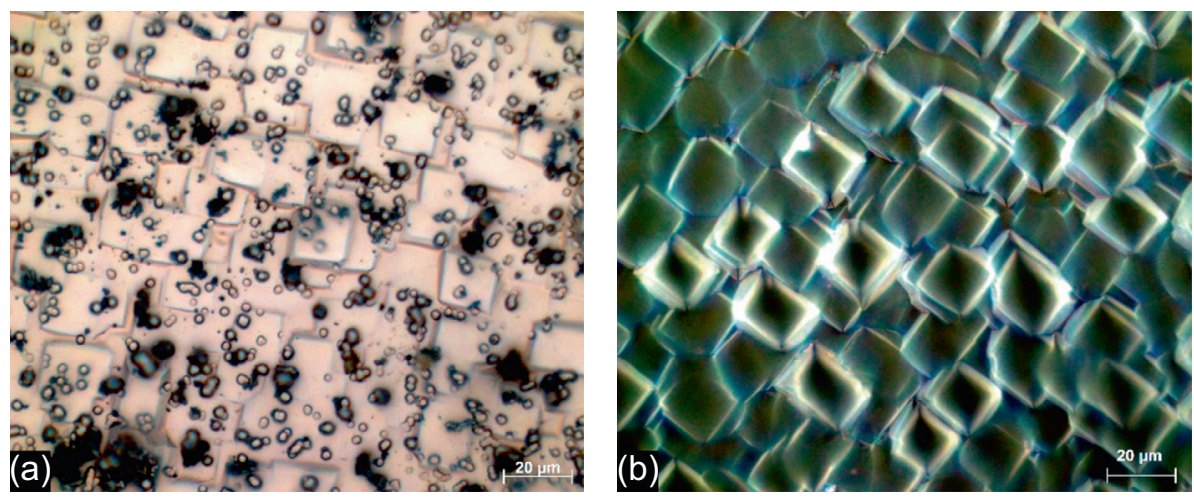

Figure 2. (a) Optical microscope image of the carbon coating and (b) the unpolished side of the Si wafer (magnification: $\times 500$ ).
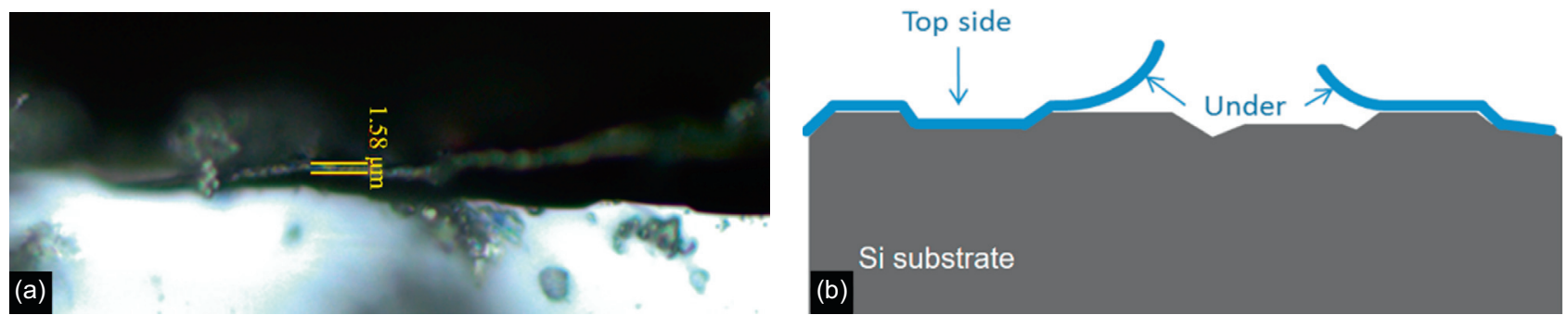

Figure 3. (a) Measuring the carbon coating thickness. (b) Schematic representation of the cross-section.
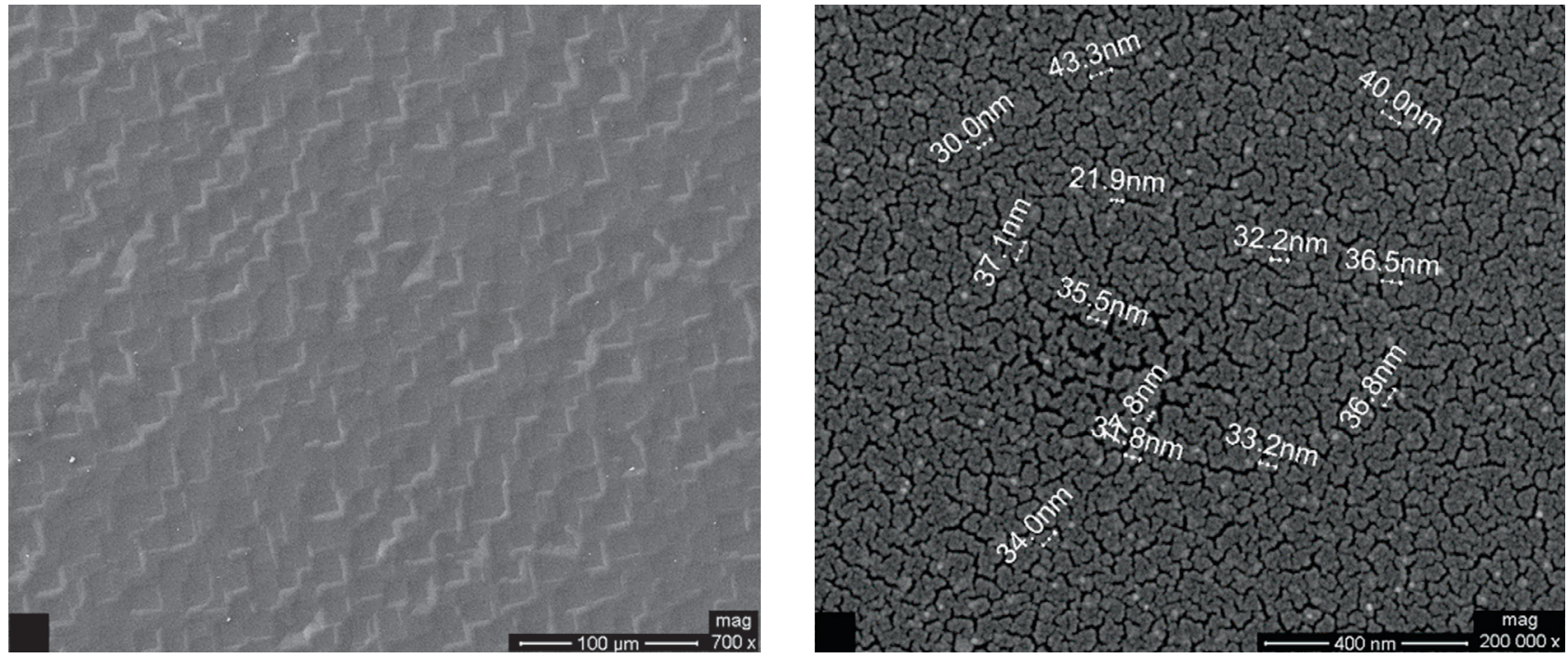

Figure 4. SEM images of the carbon coating deposited onto Si wafer.

In all nanoindentation tests a total of 10 indents are averaged to determine the mean hardness $(H)$ and elastic modulus $(E)$ values for statistical purposes, with a spacing of $50 \mu \mathrm{m}$, in a clean area environment with $45 \%$ humidity and $23{ }^{\circ} \mathrm{C}$ ambient temperature. In order to operate under closed loop load or displacement control, feedback control option was used. All nanoindentation measurements have been performed with the standard three-sided pyramidal Berkovich probe, with an average radius of curvature of about $100 \mathrm{~nm}$ [19].

Based on the half-space elastic deformation theory, $H$ and $E$ values can be extracted from theexperimental data (load displacement curves) using the Oliver-Pharr (O\&P) method [20,21]. The derived expressions for calculating the elastic modulus from indentation experiments are based on Sneddon's [22] elastic contact theory:

$$
E_{r}=\frac{S \sqrt{\pi}}{2 \beta \sqrt{A_{\mathrm{c}}}}
$$

where $S$ is the unloading stiffness (initial slope of the unloading load-displacement curve at the maximum displacement of penetration (or peak load)), $A_{\mathrm{c}}$ is the projected contact area between the tip and the substrate and $\beta$ is a constant that 

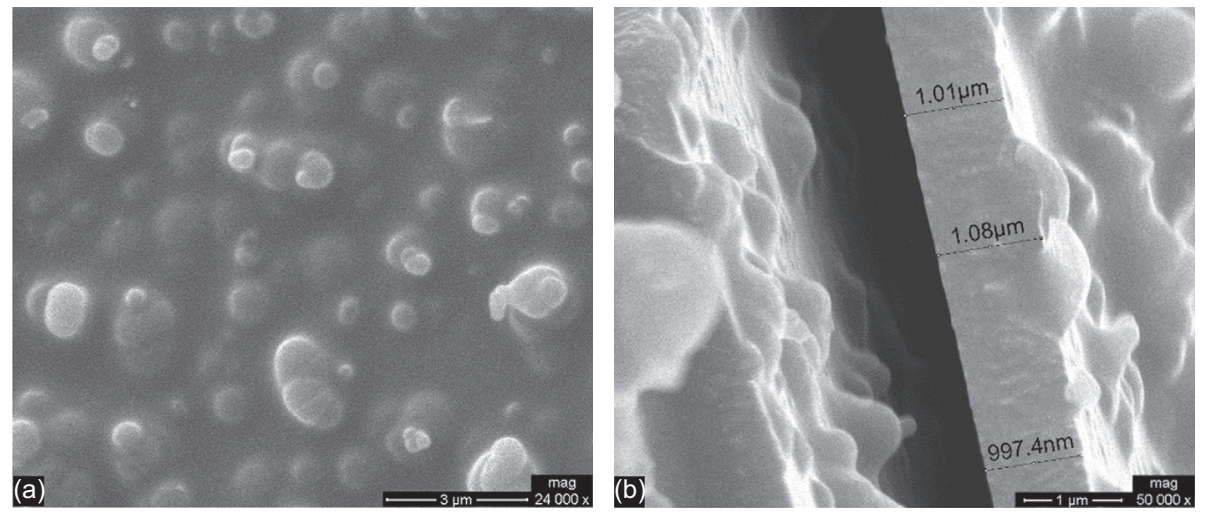

Figure 5. SEM images of the carbon coating deposited onto copper oxide nanoparticles: (a) pattern and (b) cross-section.

depends on the geometry of the indenter $(\beta=1.167$ for Berkovich tip [21, 23]). Conventional nanoindentation hardness refers to the mean contact pressure; this hardness, which is the contact hardness $\left(H_{\mathrm{c}}\right)$ is actually dependent upon the geometry of the indenter (Eqs. 2-4).

$$
H_{\mathrm{c}}=F / A
$$

where,

$$
A\left(h_{\mathrm{c}}\right)=24.5 h_{\mathrm{c}}{ }^{2}+a_{1} h_{\mathrm{c}}+a_{1 / 2} h_{\mathrm{c}}{ }^{1 / 2}+\ldots+a_{1 / 16} h_{\mathrm{c}}{ }^{1 / 16}
$$
and

$$
h_{\mathrm{c}}=h_{\mathrm{m}}-\varepsilon \frac{P_{\mathrm{m}}}{S_{\mathrm{m}}}
$$

where $h_{\mathrm{m}}$ is the total penetration displacement of the indenter at peak load, $P_{\mathrm{m}}$ is the peak load at the indenter displacement $h_{\mathrm{m}}$, and $\varepsilon$ is an indenter geometry constant, equal to 0.75 for Berkovich indenter [21-24]. Prior to indentation, the area function of the indenter tip was calibrated in a fused silica, a standard material for this purpose [25].

\section{Results and Discussion}

\subsection{Optical observations}

The obtained coating deposited on the Si wafers and the quartz tube is a shiny, metal-like film which is most of the time adhered to each substrate and difficult to be removed. The actual macroscopic condition of a representative sample is depicted in Figure 1. The coating was delaminated form the substrate in order to be investigated by various characterisation techniques.

\subsection{Surface topography/film thickness}

The coating that has been grown on the Si wafer substrate on the unpolished side, was intensively investigated via optical microscopy and SEM. The topography revealed that the coating followed the exact pattern of the Si crystals, as it is depicted in Figure 2.

The determination of the film thickness was accomplished according to the following process. The coating was delaminated from its substrate and was placed vertically under the

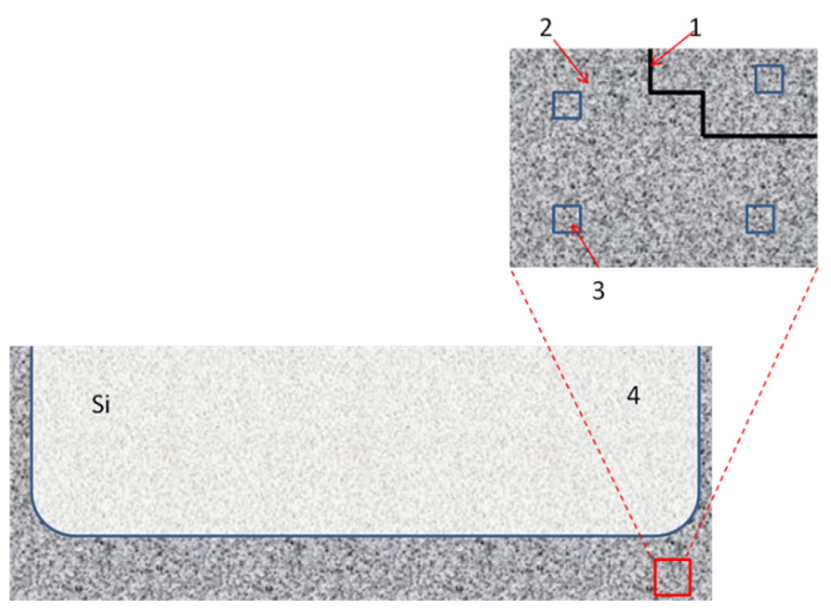

Figure 6. The spots where the Raman spectra were collected: spot no (1) region limits, spot no (2) flat surface, spot no (3) dome, spot no (4) Si wafer, spots no (5-7) flip side.

optical microscope. A thickness of about $1.58 \mu \mathrm{m}$ was measured (Figure 3).

The surface topography was examined by SEM. Figure 4 depicts the corresponding SEM images. Considering Figure 4, it may be remarked that the dome edges are smooth and round for the top surface and sharp and well defined for the other side. Moreover, several bubbles can be observed, with a diameter close to $2 \mu \mathrm{m}$. The structure of the coating consists of a consistent pattern, with a grain size ranging from $20 \mathrm{~nm}$ to $50 \mathrm{~nm}$ (Figure 4).

The SEM images of the carbon coating deposited onto copper oxide nanoparticles using acetylene are demonstrated in Figure 5. This coating followed also the pattern of the substrate, resulting in flakes that seem like wafers. The SEM image of the coating cross-section revealed that the thickness is $1 \mu \mathrm{m} \pm 10 \mathrm{~nm}$, as depicted in Figure 5 .

\subsection{Raman analysis}

The Raman spectra were collected in various spots on the sample (Figure 6 demonstrates the spots where the spectra were collected). 

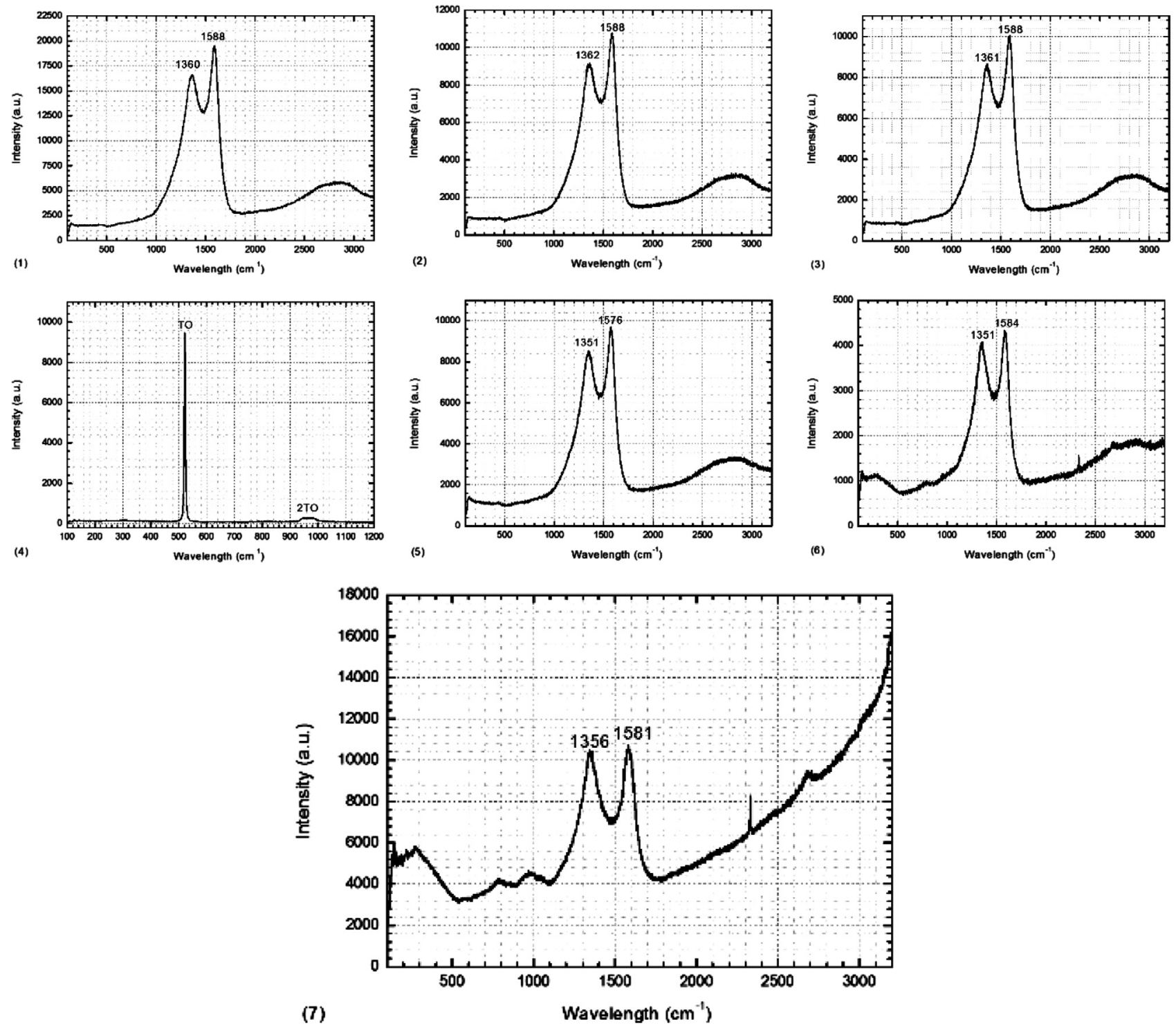

Figure 7. Raman spectrum collected spot no (1) region limits, spot no (2) flat surface, spot no (3) dome, spot no (4) Si wafer, spots no (5-7) flip side.

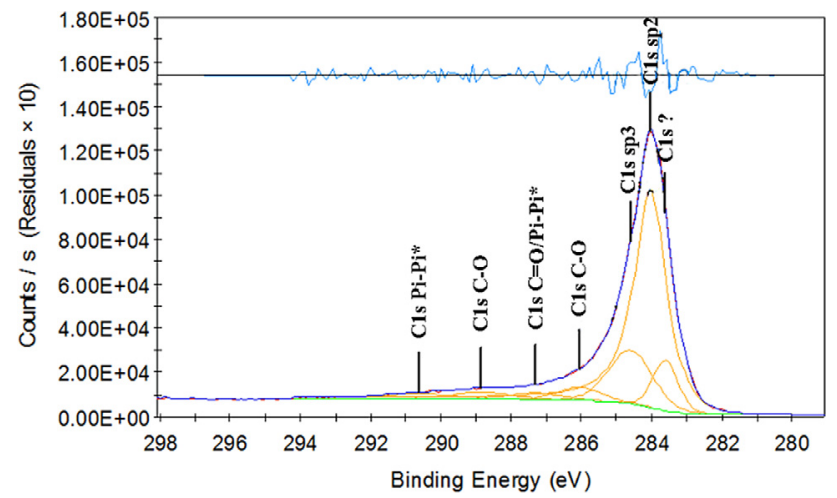

Figure 8. XPS spectrum of carbon coating (C1s).
Table 1. Atomic percentage of $s p^{2}$ and $s p^{3}$ bonds.

\begin{tabular}{lc}
\hline Bond & Atomic $\%$ \\
\hline C1s $s p^{2}$ & 62.4 \\
C1s $s p^{3}$ & 16.9 \\
O1s & 9.4 \\
Si2 $p$ & 3.7 \\
N1s & 0.4 \\
\hline
\end{tabular}

The obtained Raman spectra are displayed in Figure 7. In the Raman spectra the two main graphite peaks can be observed: The first one is the peak at $1580 \mathrm{~cm}^{-1}$ that is assigned to the in-plane vibration of the $\mathrm{C}-\mathrm{C}$ bond ( $\mathrm{G}$ band). 

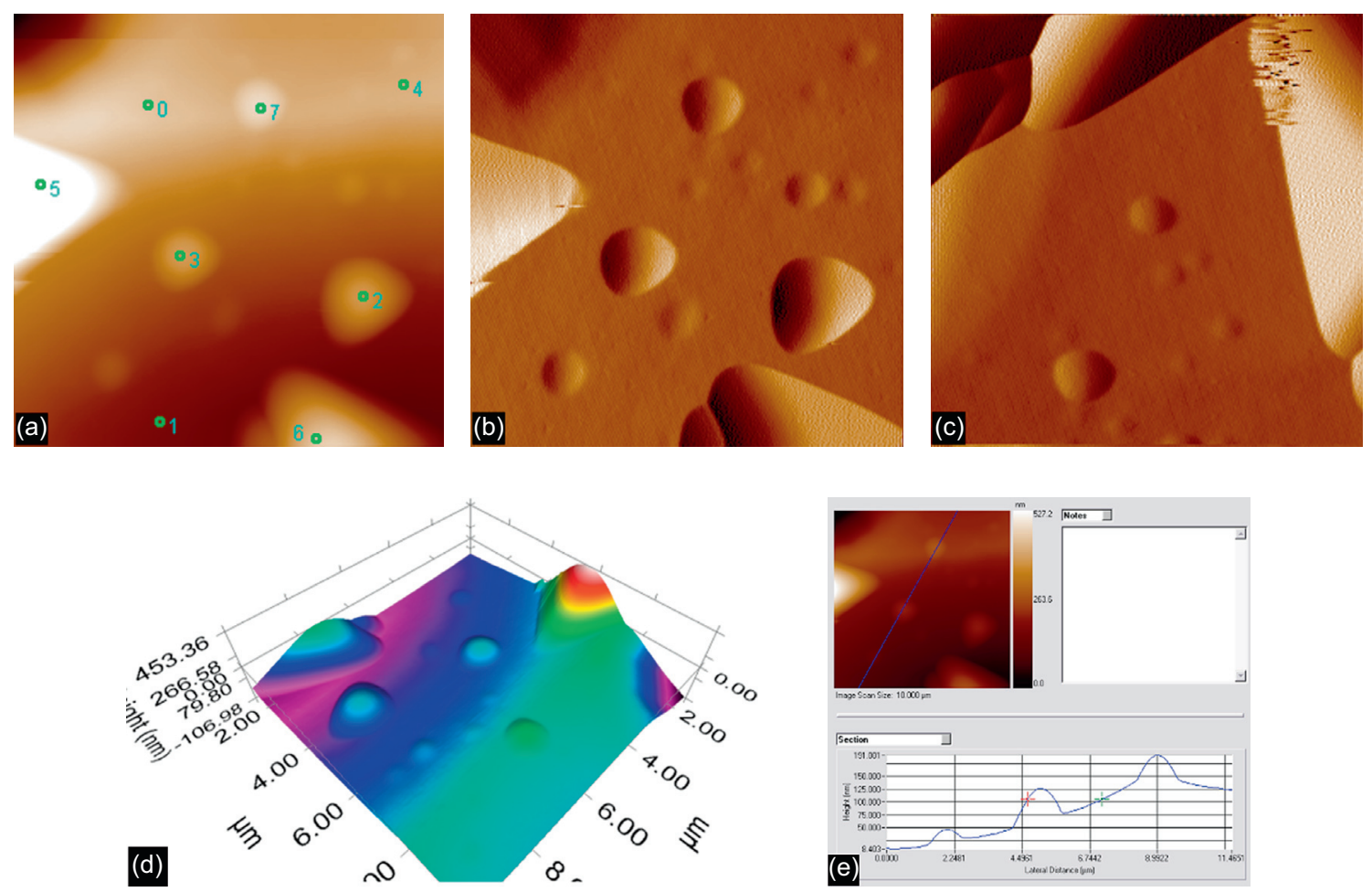

Figure 9. SPM images of the carbon coating $(10 \mu \mathrm{m} \times 10 \mu \mathrm{m})$ : (a) top-view, (b) pattern, (c) cross-section and (d, e) topography.

The presence of the shoulder around $1600 \mathrm{~cm}^{-1}$ is typical for defective graphite-like materials [26]. The second one is the peak at $\sim 1350 \mathrm{~cm}^{-1}$ (D band) due to the presence of disorder in carbon systems [27].

Additionally, the Raman spectra also exhibit a band at $\sim 2700 \mathrm{~cm}^{-1}$ that can be attributed to the overtone of the D band ( $G^{\prime \prime}$ band). The spectrum collected at spot no 4 corresponds to a typical Raman spectrum of crystalline $\mathrm{Si}$ where the TO peak at $\sim 520 \mathrm{~cm}^{-1}$, as well as the two phonon $2 \mathrm{TO}$ mode at $\sim 960 \mathrm{~cm}^{-1}$ can be clearly observed in Figure 7 .

\subsection{XPS analysis}

XPS can reveal the binding energy of the carbon atoms and discern the hybrid $s p^{3}$ and $s p^{2}$ bonds, thus is a very powerful method to evaluate the structure of amorphous carbon films without causing excessive damage to the materials [28]. From the XPS spectrum that is depicted in Figure 8, it can be observed that a high C1s peak appears in $284.08 \mathrm{eV}$, which is attributed to graphite. These results come in accordance with the atomic percentage that has been calculated for the $s p^{2}-s p^{3}$ bonds, which are illustrated in Table 1 which also includes the atomic percentage of possible impurities, such as nitrogen $(0.4 \%$ at.), which appears in the structure due to the use of nitrogen as inert gas.

\subsection{SPM imaging and nanomechanical testing}

In Figure 9, SPM imaging revealed the topography of the carbon film, indicating inhomogeneous distributions of

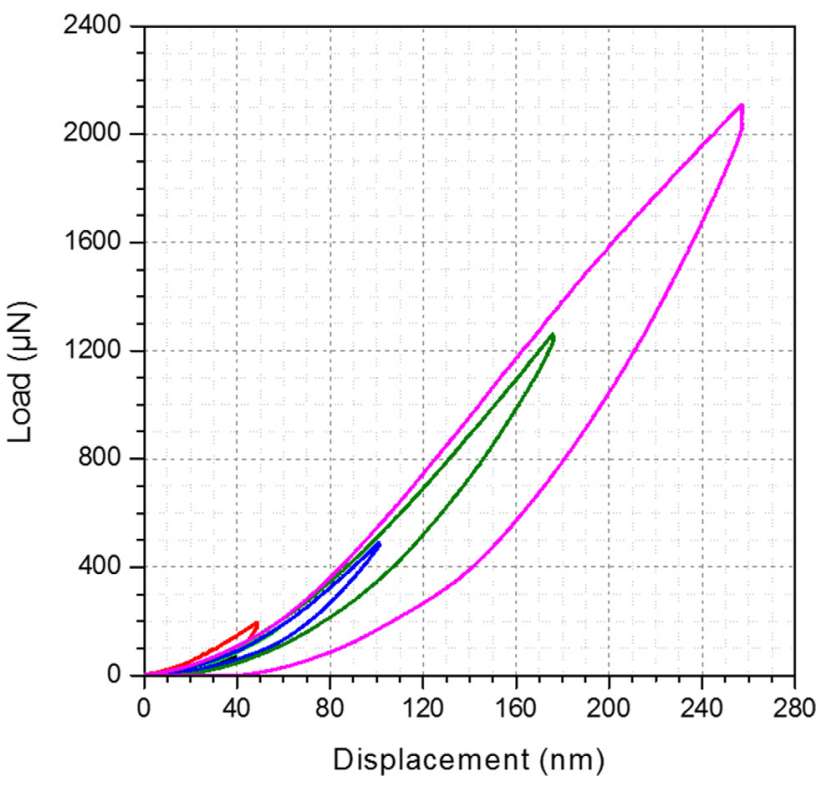

Figure 10. Load-displacement response of the film.

particle-like regions. The images of SPM were used as a selection grid to perform the nanoindentation measurements.

Typical load-displacement response of the film (Figure 10) was performed following a trapezoidal loading and unloading curve; loading and unloading segment time was $40 \mathrm{~s}$ each, and holding time was set at $3 \mathrm{~s}$.

The $H$ and $E$ modulus as a function of displacement are presented in Figure 11. Both $H$ and $E$ values were found to 
(a)

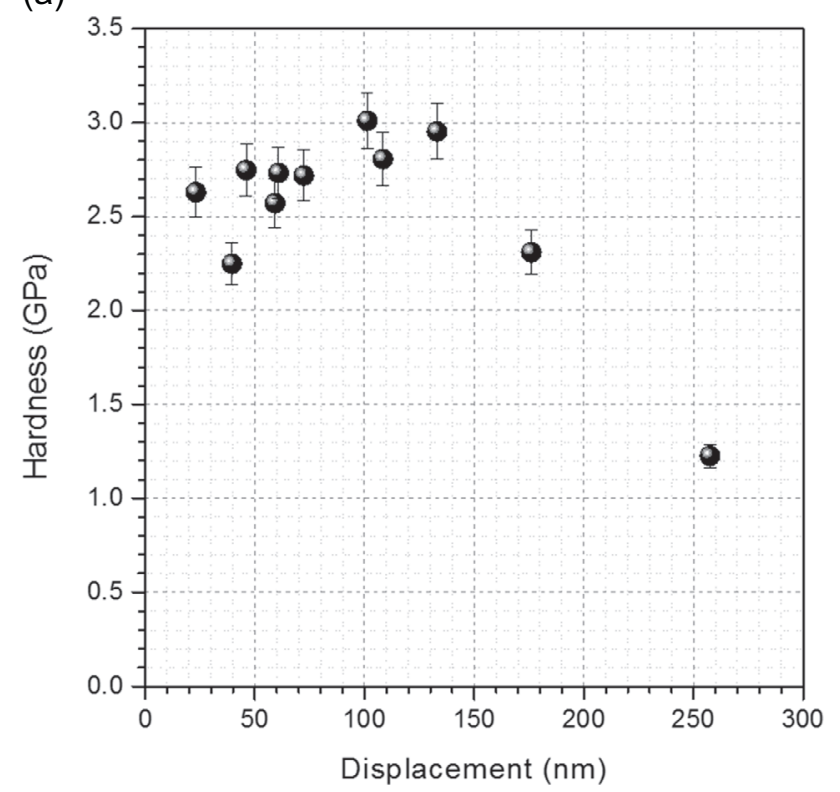

(b)

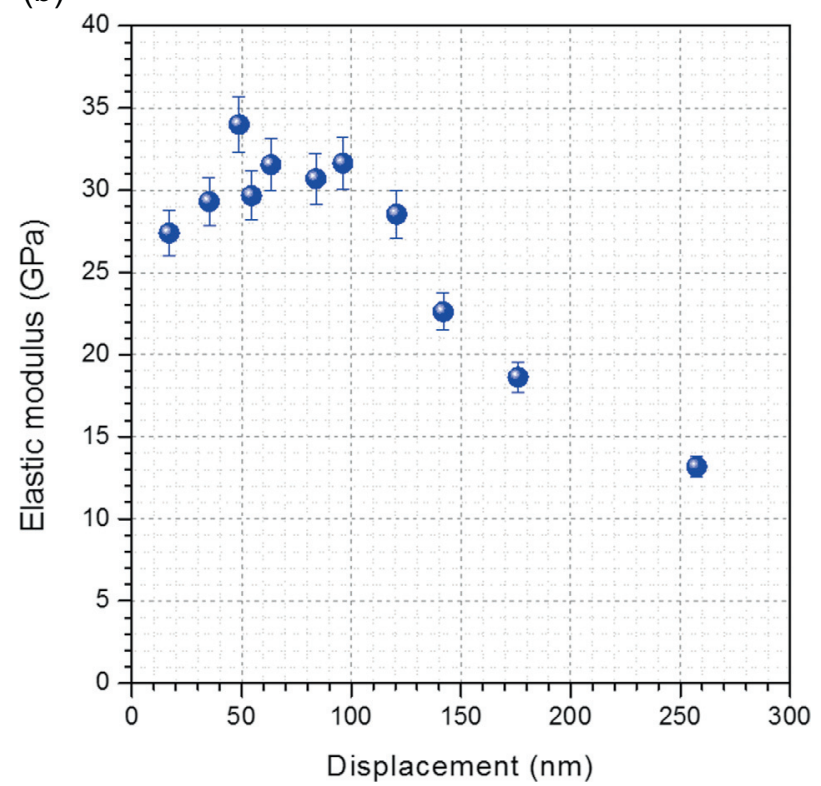

Figure 11. (a) Hardness and (b) elastic modulus of the film, as a function of displacement.

slightly deviate at surface region $(\sim 0-160 \mathrm{~nm})$, where the rule of a thumb (10\% of total film thickness) is being followed, in order to avoid the substrate effect (the film was carefully mounted and placed on polymer holder). The small deviation occurs probably due to roundness of the tip and Indentation Size Effect (ISE), tending to reach a constant value of $\sim 3$ and $\sim 33 \mathrm{GPa}$, respectively. After $160 \mathrm{~nm}$ of displacement, the substrate effect is evidenced. Due to the very low contact area between the indenter and the sample, very high stresses can be developed. The high hydrostatic pressure exerted by the surrounding material allows plastic deformation at room temperature when conventional mechanical testing only leads to fracture. It is revealed that some materials exhibit ISE, which shows an increase in hardness with decreasing applied load [29]. Apparently, the existence of ISE may hamper the accurate measurement of hardness value, and is attributed to experimental artifact, a consequence of inadequate measurement capability or presence of oxides on the surface [30]. Other explanations include indenter-specimen friction [31], and changing dislocation density for shallow indents due to the presence, for instance, of geometrically necessary dislocations [32]. The Berkovich indenter generates dislocations organized in a quite complex way during a nanoindentation test, even for very low deformations [33], making difficult the formulation for the stress field generated, even during an elastic deformation, as well as its modelling. Most of the dislocations stay generally confined around the residual imprint in a dense structure $[34,35]$ with many dislocation interactions [36].

The ratio of hardness/elastic $(H / E)$ modulus is of significant interest in tribology. Higher stresses are expected in high $H / E$, hard materials, and high stress concentrations develop towards the indenter tip, whereas in the case of low $H / E$, soft materials, the stresses are lower and are distributed more

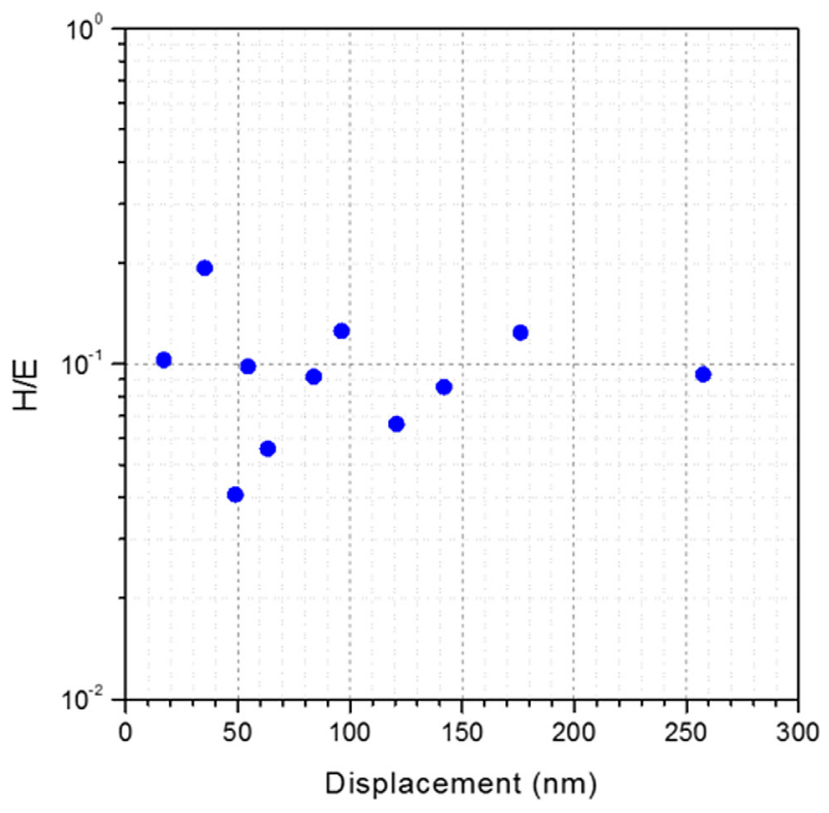

Figure 12. Wear resistance index $(H / E)$ of the film, as a function of displacement.

evenly across the cross-section of the material [37, 38]. The high ratio of $H / E$ is indicative of the good wear resistance (Figure 12) in a disparate range of materials [37, 39]: ceramic, metallic and polymeric (e.g. c-BN, tool steel and nylon, respectively), which are equally effective in resisting attrition for their particular intended application. As evidenced in Figure 14, at lower elastic recovery regions, $H / E$ increases; the deformation exhibits a linear trend (noted with dashed line). 


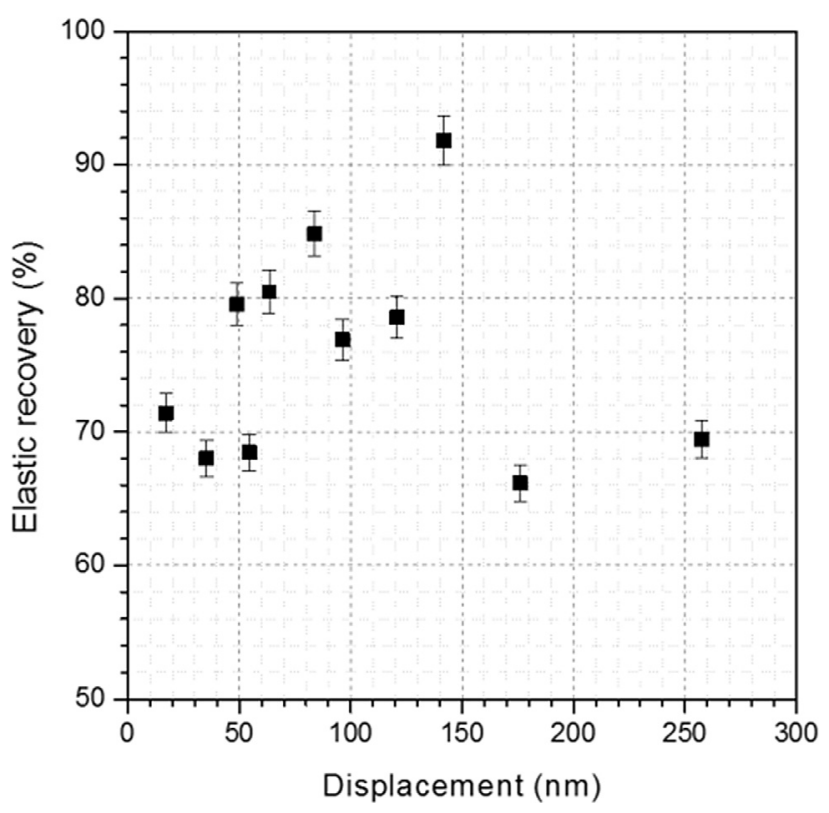

Figure 13. Elastic recovery of the film, as a function of displacement.

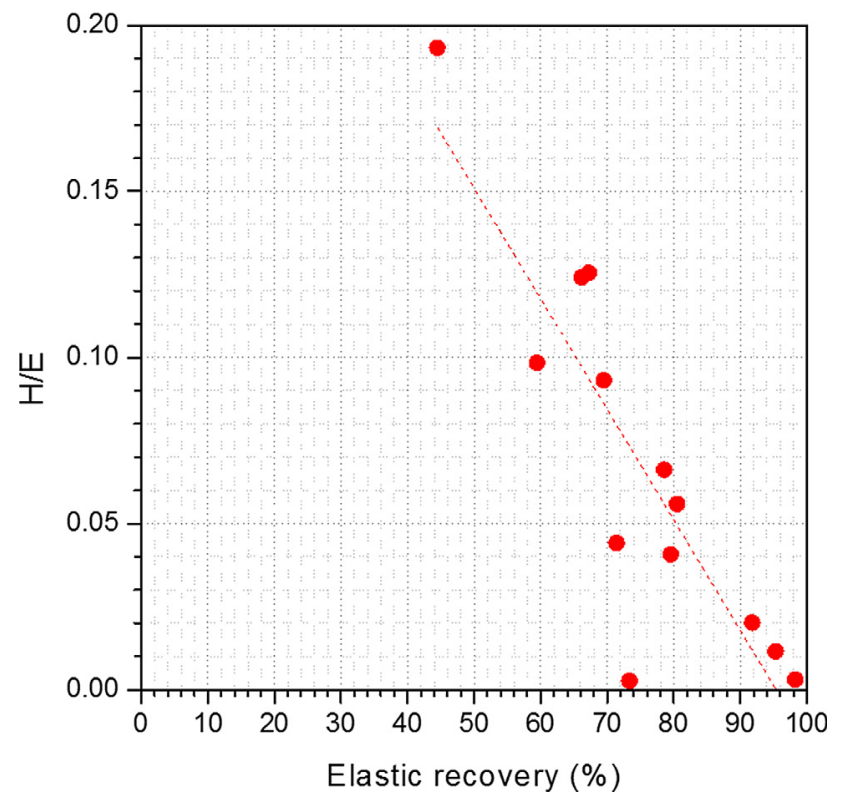

Figure 14. Wear resistance index $(H / E)$ of the film, as a function of elastic recovery.

\section{Conclusions}

A carbon film that appears during the CNT synthesis, using a T-CVD system was identified using microscopy and spectroscopy characterizations. The carbon film was noticed on different substrates inside the reactor and taking into consideration the aforementioned characterizations, it may be remarked that the obtained film is classified as an amorphous carbon film. The topography revealed that the coating followed the exact pattern of the Si crystals or the copper oxide nanoparticles, while determination of the film thickness was accomplished in both cases and gave a film thickness of $1 \mu \mathrm{m}$. The interpretation of Raman and XRS (X-ray Raman spectroscopy) results, revealed that the carbon film contains a high percentage of $s p^{2}$ bonds, which comes in accordance with the macroscopic properties of the film (opacity and metallic luster). Presence of oxygen was also confirmed by the XPS, which can be attributed to the oxygen contained in camphor, as carbon precursor. Through nanoindentation, hardness and elastic modulus together with wear resistance index $(H / E)$ were evaluated. After $160 \mathrm{~nm}$ of displacement, the substrate effect was evidenced. At lower elastic recovery regions, $H / E$ increases, while the deformation exhibits a linear trend. The above results, draw the conclusion that the amorphous carbon film can be regarded as a soft film. Further investigation on the process parameters can lead to the exclusive synthesis of this coating, on selected ceramic substrates, to change their surface properties, such as their hydrophobicity and the reduction of their porosity, for sealant applications. The thorough characterization of the produced carbon film, in combination with the control of the synthesis parameters, gives the opportunity to manufacture amorphous carbon films in production environments using CVD systems. A careful control of the growth parameters and a selection of different substrates can enhance the application fields of such materials.

Acknowledgements. This material is based upon work supported by the EU FP7 Project "Low-toxic cost-efficient environment-friendly antifouling materials" (BYEFOULING) under Grant Agreement No. 612717.

\section{References}

1. J. Robertson, Diamond-like amorphous carbon, Materials Science and Engineering: R: Reports 37 (2002) 129-281.

2. J.-M. Bonard, Carbon nanostructures by hot filament chemical vapor deposition: growth, properties, applications, Thin Solid Films 501 (2006) 8-14.

3. S. Lee, J. Won, J. Choi, S. Jang, Y. Jee, H. Lee, D. Byun, Preparation and analysis of amorphous carbon films deposited from (c6h12)/ar/he chemistry for application as the dry etch hard mask in the semiconductor manufacturing process, Thin Solid Films 519 (2011) 6737-6740.

4. D. Zhu, Y. Liu, L. Yuan, Y. Liu, X. Li, L. Yi, H. Wei, K. Yao, Controllable synthesis of large-area free-standing amorphous carbon films and their potential application in supercapacitors, RSC Advances 4 (2014) 63734-63740.

5. J.V.S. Moreira, P.W. May, E.J. Corat, A.C. Peterlevitz, R.A. Pinheiro, H. Zanin, Diamond and carbon nanotube composites for supercapacitor devices, Journal of Electronic Materials 46 (2016) 929-935.

6. D.S. da Silva, A.D.S. Côrtes, M.H. Oliveira, E.F. Motta, G.A. Viana, P.R. Mei, F.C. Marques, Application of amorphous carbon based materials as antireflective coatings on crystalline silicon solar cells, Journal of Applied Physics 110 (2011) 043510.

7. J.P. Sullivan, T.A. Friedmann, K. Hjort, Diamond and amorphous carbon mems, MRS Bulletin 26 (2011) 309-311.

8. M. Santos, M.M.M. Bilek, S.G. Wise, Plasma-synthesised carbon-based coatings for cardiovascular applications, Biosurface and Biotribology 1 (2015) 146-160. 
9. S. Mitura, E. Mitura, A. Mitura, Manufacture of amorphous carbon layers by r.f. dense plasma CVD, Diamond and Related Materials 4 (1995) 302-303.

10. K.O. Park, B.D. An, S.J. Lee, Method of forming amorphous carbon film and method of manufacturing semiconductor device using the same, Patent: US20080293248 (2008).

11. S. Hidzhazi, Nano-manufacturing and product design: practical solutions to current manufacturing challenges, International Journal of Interdisciplinary Research and Innovations 3 (2015) 66-72.

12. A. Eatemadi, H. Daraee, H. Karimkhanloo, M. Kouhi, N. Zarghami, A. Akbarzadeh, M. Abasi, Y. Hanifehpour, S.W. Joo, Carbon nanotubes: properties, synthesis, purification, and medical applications, Nanoscale Research Letters 9 (2014) 393.

13. S. Jae Keun, K. Ki-han, K. Jaekwang, L. Yu sung, K. Eun Kyu, L. Jae-Hyeoung, C. Won Seok, Growth of metal-free carbon nanotubes with amorphous carbon catalyst layer on glass substrates by microwave plasma enhanced chemical vapor deposition, in: P.K. Chu (Ed.), Nanoelectronics Conference (INEC) 3rd International, IEEE Catalogue Number: CFP10625, ISBN: 978-1-4244-3544-9, Library of Congress: 2008911110 , pp. $79-80$.

14. A. Bachmatiuk, J. Boeckl, H. Smith, I. Ibrahim, T. Gemming, S. Oswald, W. Kazmierczak, D. Makarov, O.G. Schmidt, J. Eckert, L. Fu, M.H. Rummeli, Vertical graphene growth from amorphous carbon films using oxidizing gases, The Journal of Physical Chemistry C 119 (2015) 17965-17970.

15. C. Yang, P. Wu, W. Gan, M. Habib, W. Xu, Q. Fang, L. Song, Low temperature CVD growth of ultrathin carbon films, AIP Advances 6 (2016) 055310.

16. A. Barreiro, F. Börrnert, S.M. Avdoshenko, B. Rellinghaus, G. Cuniberti, M.H. Rümmeli, L.M.K. Vandersypen, Understanding the catalyst-free transformation of amorphous carbon into graphene by current-induced annealing, Scientific Reports 3 (2013), 1-6.

17. N. Larouche, B.L. Stansfield, Classifying nanostructured carbons using graphitic indices derived from Raman spectra, Carbon 48 (2010) 620-629.

18. C.F. Cullis, N.H. Franklin, The pyrolysis of acetylene at temperatures from 500 to $1000{ }^{\circ} \mathrm{C}$, Proceedings of the Royal Society A: Mathematical, Physical and Engineering Sciences 280 (1964) 139-152.

19. C. Charitidis, E. Koumoulos, D. Dragatogiannis, Nanotribological behavior of carbon based thin films: friction and lubricity mechanisms at the nanoscale, Lubricants 2 (2013) 22-47.

20. E.P. Koumoulos, C.A. Charitidis, D.P. Papageorgiou, A.G. Papathanasiou, A.G. Boudouvis, Nanomechanical and nanotribological properties of hydrophobic fluorocarbon dielectric coating on tetraethoxysilane for electrowetting applications, Surface and Coatings Technology 206 (2012) 3823-3831.

21. W.C. Oliver, G.M. Pharr, An improved technique for determining hardness and elastic modulus using load and displacement sensing indentation experiments, Journal of Materials Research 7 (2011) 1564-1583.

22. I.N. Sneddon, Boussinesq's problem for a rigid cone, Mathematical Proceedings of the Cambridge Philosophical Society 44 (2008) 492.
23. L. Huang, J. Lu, M. Troyon, Nanomechanical properties of nanostructured titanium prepared by smat, Surface and Coatings Technology 201 (2006) 208-213.

24. R.B. King, Elastic analysis of some punch problems for a layered medium, International Journal of Solids and Structures 23 (1987) 1657-1664.

25. H. Bei, E.P. George, J.L. Hay, G.M. Pharr, Influence of indenter tip geometry on elastic deformation during nanoindentation, Physical Review Letters 95 (2005) 045501.

26. L. Bokobza, J. Zhang, Raman spectroscopic characterization of multiwall carbon nanotubes and of composites, Express Polymer Letters 6 (2012) 601-608.

27. A.C. Ferrari, Raman spectroscopy of graphene and graphite: disorder, electron-phonon coupling, doping and nonadiabatic effects, Solid State Communications 143 (2007) 47-57.

28. P.K. Chu, L. Li, Characterization of amorphous and nanocrystalline carbon films, Materials Chemistry and Physics 96 (2006) 253-277.

29. E.P. Koumoulos, P. Jagdale, I.A. Kartsonakis, M. Giorcelli, A. Tagliaferro, C.A. Charitidis, Carbon nanotube/polymer nanocomposites: a study on mechanical integrity through nanoindentation, Polymer Composites 36 (2015) 1432-1446.

30. H. Li, A. Ghosh, Y.H. Han, R.C. Bradt, The frictional component of the indentation size effect in low load microhardness testing, Journal of Materials Research 8 (2011) 1028-1032.

31. Q. Ma, D.R. Clarke, Size dependent hardness of silver single crystals, Journal of Materials Research 10 (2011) 853-863.

32. Y. Gaillard, C. Tromas, J. Woirgard, Quantitative analysis of dislocation pile-ups nucleated during nanoindentation in mgo, Acta Materialia 54 (2006) 1409-1417.

33. E.P. Koumoulos, C.A. Charitidis, Surface analysis and mechanical behaviour mapping of vertically aligned cnt forest array through nanoindentation, Applied Surface Science 396 (2017), 681-687.

34. Y.L. Chiu, A.H.W. Ngan, Time-dependent characteristics of incipient plasticity in nanoindentation of a $\mathrm{Ni}_{3} \mathrm{Al}$ single crystal, Acta Materialia 50 (2002) 1599-1611.

35. E.P. Koumoulos, T. Parousis, A.F.A. Trompeta, I.A. Kartsonakis, C.A. Charitidis, Investigation of mwent addition into poly-dimethylsiloxane-based coatings, Plastics, Rubber and Composites 45 (2016) 106-117.

36. T. Schlagradl, R. Schneider, G. Posch, R. Schnitzer, Investigation of the hardness-toughness relationship of a welded joint after different heat treatment cycles, Welding in the World 57 (2012) 113-121.

37. A. Leyland, A. Matthews, Design criteria for wear-resistant nanostructured and glassy-metal coatings, Surface and Coatings Technology 177-178 (2004) 317-324.

38. Y.-T. Cheng, C.-M. Cheng, What is indentation hardness?, Surface and Coatings Technology 133-134 (2000) 417-424.

39. A. Leyland, A. Matthews, Optimization of nanostructured tribological coatings, in: A. Cavaleiro, J.Th.M. de Hosson (Eds.), Nanostructured Coating, 1st edn., Springer Science + Business Media, LLC, 2006, pp. 511-538. 